\title{
Seroepidemiological Study of Measles Virus among Young Adults: Response to Revaccination
}

\author{
Arwa H. Al-Hamdani* \\ Received 24, November, 2013 \\ Accepted 17, February, 2014
}

\begin{abstract}
:
The 2011 Iraqi Measles Control Campaign had as its aim to improve immunization coverage among young adults' 17-24years and, in the longer term, prevention of measles epidemics. The aim of the study is estimation measles vaccination and revaccination efficacy by evaluate the seroprevalence of antibody and response to reimmunization in young adults. A previously vaccinated 189 young adult medical students were tested for measles specific IgG \&IgM pre and post catch-up revaccination by using commercially available ELISA. To assess the seroprevalence of antibody response pre measles reimmunization in previously vaccinated young adults; 45 were found to be seronegative. To differentiate between primary and secondary vaccine failure, anti- measles IgM and IgG titers were assessed again 2-4 weeks after revaccination in 189 seronegative participants [45 seronegative, 144 seropositive individuals: 29 seronegative participants responded to revaccination anamnestically $(\mathrm{P}<0.001)$ and developed immunity; 7 also showed IgM response (probably primary vaccine failure); 144 seropositive participants remained seroprotected without significant increase in antibody titer $(\mathrm{P}=0.577)$. Primary vaccine failure was fond to be $3.7 \%$; while secondary vaccine failure was $11.6 \%$.After revaccination, $85.1 \%$ were seroprotected. This study demonstrates the waning measles immunity in young adults in Iraqi medical students, which poses the potential risk of transmission of measles. Periodic Serological surveillance and revaccination of susceptible young adults are recommended.
\end{abstract}

\section{Key words: Measles, Seroprevalence, Revaccination}

\section{Introduction:}

Measles is a highly contagious viral illness that has fortunately been fairly well-controlled through measles vaccination plans. Measles vaccine is inducing long -term and probably lifelong immunity in most individuals [1]. Although effective, measles vaccination with only one dose provides just $95 \%$ protection against measles. This led to the recommendation of a second dose for children between 5-19 years of age, to ensure protection for those who had not developed immunity from the first dose [2].A second, booster dose helps to improve the effectiveness of the measles vaccine to over $99 \%$. The routine measles vaccination plan calls for kids to get the first MMR (Measles, Mumps, Rubella) vaccine when they are 12 to 15 months old and the MMR booster when they are 4 to 6 years old but didn't become routine until 1990[3].

During 1980, it was reported 26542, measles cases in Iraq. This figure decreased to 3045 and 726 , cases during 1990 and 2000, due to successful expanded program in immunization (EPI) in Iraq, but in

*Lecturer, Department of Microbiology/College of Medicine, Al-Mustansiriyah

University, 
2004, $2005 \& 2006$, there was some increase, then decline in measles cases (9082,908\&474), respectively

[4].Significant progress has been made in the Eastern Mediterranean Region (EMR) toward measles elimination, through increasing coverage with a routine Measles -containing vaccine (MCV) and implementation of catchup and follow-up measles supplemental immunization activities (SIAs) in EMR countries. The number of confirmed measles cases has decreased dramatically from 89,518 in 1998 to 12,186 in $2008 \quad(86 \%$ decrease), and the EMR achieved a 93\% reduction in estimated measles mortality during 2000-2008-the largest percentage reduction among all WHO regions, accounting for $17 \%$ of the global reduction in measles mortality [5]. Despite these successes, in recent years, there has been a resurgence of measles virus circulation and outbreaks in a number of countries, including a nationwide epidemic in Iraq during 2008-2009was a clear indication of serious problems in routine and (SIA).Due to lack of funding WHO could not support the requested support to conduct (SIA) \& too weak health infrastructure [6]. Records indicate that around 27,000 Iraqi children were infected with measles between 2008 and 2010.As a result of ceaseless efforts the ministry made to fight this disease, which is pandemic in Iraq, the incidence of the disease was only one case recorded during 2012, which is a major accomplishment [7].A low vaccine failure rate of $66.1 \%$ and effectiveness of $90.03 \%$ were estimated in Iraq for the years 2005 to 2010 in a study which concluded that measles continues to be an important cause of morbidity in Iraq. Improvements in vaccine coverage, proper vaccine handling, and prompt reporting of suspected cases are all necessary to eliminate measles from Iraq [8].The primary reason for continuing high childhood morbidity is the failure to deliver at least one dose of measles vaccine to all infants [9].

Vaccine failure remains a major obstacle that must be overcome before measles can be controlled [10]. Primary vaccine failure (PVF) is failure of immediate seroconversion, with a documented lack of detectable specific antibody. Administration of a second dose of vaccine results in a high proportion undergoing a primary antibody response, with an initial IgM response followed by IgG seroconversion. Secondary vaccine failure (SVF) results when there is initial documented seroconversion in response to vaccination followed by loss of protection, usually linked to waning serum antibody levels [11]. On exposure to measles virus, an individual may contract the virus and only show anamnestic type of antibody response, or may become ill. Following a second dose of vaccination, a large boost in $\mathrm{IgG}$ antibody levels generally occurs, with little or no IgM response [11].Around 95\%-98\% of individuals who receive a single dose of measles vaccine after 12 months of age develop measles antibodies [12]. The measles vaccine is, however, less immunogenic in the presence of maternal antibodies before 12 months of age [11]. The Immunization policy in Iraq follows the World Health Organization's recommendation for developing countries, and includes monocomponent vaccine against measles at 9 months of age [13]. In addition, vaccination against measles has been recommended for children aged 12-15 months in Iraq which was started in 1986 as a single measles antigen vaccine, then at 1988 MMR was introduced [14]. Excellent seroconversion rated $(96.8 \%)$ was 
obtained among children in response to MMR vaccination, who's received mono-valent measles vaccine during their first year of age, in contrast moderate seroconvertion rate was obtained after measles live vaccine, and approximately quarter remained seronegative which represents PVF to measles vaccine [15]. After implementing the 2-dose vaccination schedule with high levels of coverage (>95\%), between 1988 and 1998 the incidence of measles decreased markedly in Iraq. During recent years, however, the incidence of the disease has been increasing, with some incidences in previously vaccinated individuals among all age groups, mainly adolescents and young adults [16].

This study was designed to evaluate the IgM and IgG antibody responses to reimmunization with measlescontaining vaccine (MCV) to differentiate PVF from SVF especially in young adults who had been vaccinated against measles according to the vaccination schedule in Iraq[On the basis of WHO recommendations, MCV has been administered in the EMR to children at 9 months of age in countries with high measles incidence and to children 12 months of age in countries with low measles incidence and a low risk of measles infection among infants [17].

\section{Materials and Methods: \\ Study subject;}

This study was carried out from September 2011 to February 2012. Healthy volunteers [with no history of medical problems (e.g. acute respiratory infection, febrile illnesses, and skin rash), physician-diagnosed measles or chronic illness] between 17 and 24 years of age were recruited from the student population at AlMustansiriyah University/Medical College. Written informed consent was obtained from all participants with vaccine histories. The protocol was reviewed and approved by the Medical Ethics Committee of the university.

\section{Blood sampling;}

for initial screening, $5 \mathrm{~mL}$ of venous blood was drawn from each participant by the researcher. Samples were taken during September 2011October 2011 and for the second phase during January 2012-February 2012. Sera were stored at $-20^{\circ} \mathrm{C}$ until assayed. Measles Catch-up campaign vaccination programme in Iraq (October 2011), all individuals (17-24) years of age were vaccinated with measles virus-containing vaccine (Rouvax brand Sanofi Pasteur-MSD in Lyon, France), i.e. all those in the study sample were revaccinated after the first blood sampling. Sera were obtained 2-4 weeks after revaccination to determine the IgM and IgG antibody responses to revaccination.

\section{Serologic assay}

Measles IgG and IgM antibodies were detected by enzyme-linked immunosorbent assay (Measles IgMELISA and measles IgG-ELISA, IBL, Hamburg). The tests were performed in the Virology Lab. /Al-Yarmook Teaching Hospital, Ministry of Health according to the manufacturer's instructions. Quantitative antibody titers $<10 \mathrm{IU} / \mathrm{mL}$ were reported as negative and $\geq 10 \mathrm{IU} / \mathrm{mL}$ as positive. All sera (samples obtained before and after revaccination) were tested for IgG measles-specific antibodies; IgM was measured only in blood samples taken after revaccination to differentiate primary and secondary response types.

\section{Statistical analysis}

Data were analyzed as ordinal and continuous variables. Antibody levels werelog-transformed for calculation of geometric mean antibody 
concentrations. The student t-test was used to compare the mean values in the pre- and post-vaccination groups and Pearson Chi-square test at 0.05 levelsto test the significances. All statistical analysis was performed using SPSS, version11.

\section{Results:}

A total of 189 young adults, mean age $22.3 \pm 3.3$ years (range 17-24 years), were enrolled in a measles seroprevalence study underwent serological screening for measles specific IgG antibody. All of these individuals had previously received measles vaccine, 2 doses (at 9 months and 15 months) in 110 participants (age range 17-21 years) and one dose (after 12 months) in 79 participants (aged 22 years and older) as part of the routine measles vaccination schedule in Iraq.

The study revealed that 45 $(23.8 \%)$ participants were serologically negative for measles antibody, geometric mean concentration $4.1 \pm 2.6$ $\mathrm{IU} / \mathrm{ml}$ (mean age $20.3 \pm 2.8$ years) and 144 (76.2\%) were seropositive, geometric mean concentration $23.9 \pm 37.2$ (mean age 21.0 \pm 2.6 years) (Table 1).

All the 189 individuals were revaccinated (as part of catch-up program, October 2011) and the follow up for their measles sero-prevalence post vaccination showed 45 seronegative and 144 seropositive individuals (Table 1). Twenty-nine of revaccinated seronegative individuals $(64.4 \%)$ responded to revaccinationand showed significant increase in the levels of measles antibody $(\mathrm{P}<0.001)$ (Table 2); 7(15.6\%) also showed IgM response (Probably primary vaccine failure). The revaccinated seroprotected individuals; remained seropositive with no statistically significant increase in $\mathrm{IgG}$ measles $\operatorname{antibody}(\mathrm{p}=0.577)$. No $\operatorname{IgM}$ response was detected in them.

As shown in Table 1 comparison of the seropositivity rates and responses to revaccination in two age groups; 17-21 years (previously received 2 doses of measles vaccine) and 21 years and older (previously received 1 dose of measles vaccine were found to be; 28 (25.45\%) of 110 individuals in the younger group and $17(21.5 \%)$ of 79 in older age group were seronegative. Table 3 showed $51.7 \%$ of the revaccinated participants enrolled in younger ( 2 dose) group responded to revaccination compared to $87.5 \%$ in the older (single dose) group with significant difference $(\mathrm{p}=0.016)$.

Based on our results, extrapolations can be made regarding PVF, SVF and efficacy of initial vaccination and revaccination programs [18]. Thereafter PVF was estimated at $3.7 \%$ $(45 \times 100 \times 7) /(189 \times 45)$ and SVF at $11.6 \%(45 \times 22 \times 100) /(189 \times 45)$ and total vaccination and revaccination efficacy was estimated at $85.1 \%[144+(45 \times 29) /(189 \times 9)]$,so

community immunity on $95 \%$ vaccination coverage would be $80.9 \%[(85.1 \times 95) / 100]$, where $189=$ total no. participants in primary screening, $45=$ no. seronegative in primary screening, $9=$ no. seronegtive after revaccination, $29=$ no. responding to revaccination $(\operatorname{IgM}$ and $\operatorname{IgG}), 22=$ no. seronegative responding with $\mathrm{IgG}$ response only (probably SVF), 7=no. seronegative responding with $\operatorname{IgM}$ (probably PVF) and 144=no. immune in primary screening. 
Table1: The seroprevalence status of young adults beforemeasles revaccination.

\begin{tabular}{|c|c|c|c|c|c|c|}
\hline Measles seroprevalence & \multicolumn{2}{|c|}{$\begin{array}{c}\text { Total } \\
(\mathbf{n = 1 8 9})\end{array}$} & \multicolumn{2}{c|}{$\begin{array}{c}\mathbf{1 7 - 2 1} \text { years } \\
(\mathbf{n = 1 1 0})\end{array}$} & \multicolumn{2}{c|}{$\begin{array}{c}\text { 22 years \& older } \\
(\mathbf{n = 7 9})\end{array}$} \\
\hline & No. & $\%$ & No. & $\%$ & No. & \% \\
\hline Antibody positive* & 144 & 76.2 & 82 & 74.55 & 62 & $\mathbf{7 8 . 5}$ \\
\hline Antibody negative** & 45 & 23.8 & 28 & 25.45 & 17 & $\mathbf{2 1 . 5}$ \\
\hline GMC \pm SD (IU/mL) & \multicolumn{2}{|c|}{$23.9 \pm 37.2$} & \multicolumn{2}{c|}{$22.4 \pm 37.2$} & $\mathbf{2 6 . 4 \pm 3 7 . 3}$ \\
\hline
\end{tabular}

Table 2: The comparison of response to revaccination between seronegative and seroprotected individuals.

\begin{tabular}{|c|c|}
\hline & Seronegative (n=45) \\
\hline IgG response & $22(48.8 \%)$ \\
\hline IgM response & $7(15.6 \%)$ \\
\hline IgG \& IgM response & $29(64.4 \%)$ \\
\hline GMC Pre vaccination (Mean \pm SD) & $4.1 \pm 2.6$ \\
\hline GMC Post vaccination (Mean \pm SD) & $37.9 \pm 42.3$ \\
\hline \multicolumn{2}{|c|}{ GMC=Geometric mean antibody concentration \pm SD (Standard deviation) } \\
\hline
\end{tabular}

Table 3: Comparison of response to revaccination in seronegative individuals according to number of doses of measles vaccine originally given.

\begin{tabular}{|c|c|c|}
\hline Doses & Total $(\mathbf{n = 4 5})$ & Responded No.(\%) \\
\hline One Measles dose & 16 & $14(87.5 \% *)$ \\
\hline Two Measles doses & 29 & $15(51.7 \%)$ \\
\hline \multicolumn{2}{|c|}{ P value=0.016* (Significant using Pearson Chi-square test at 0.05 level) } \\
\hline
\end{tabular}

\section{Discussion:}

This was the first attempt to our knowledge of mass catch-up campaign of young adults in Iraq which were at October 2011. Serological surveys should be conducted to characterize measles seroepidemiology prior and post to that mass vaccination. The increase of vaccination rates means that fewer children will be vulnerable to vaccine preventable diseases such as measles, which will invariably result in a drop in the infant mortality and morbidity rates [19].

The presenting results showed measurement of anti-measles $\mathrm{IgG}$ antibody titers 17 to 24 years after 1 or 2 doses of measles vaccination to determine the proportion of seropositivity. Of the studied population $23.8 \%$ were seronegative, and there was no difference between those who had 1 dose of vaccine and those who had 2 doses (Table1).Reinfection and disease seem to occur in individuals who have previously had a measles immunization and when the titer has fallen below a critical level [16, 20].Our results in Table- 3 were found to be consistent with previous results; children vaccinated twice have better protection than single dose recipients $[11,13]$ and inconsistent with others elsewhere;additional doses of vaccine could not boost the antibodies to a satisfactory level, and that any boosting that did take place was only short-lived [11, 13].

Although the waning immunity after immunization may be a factor in previously immunized children seem to occur in people in whom response to the vaccine was inadequate [i.e. Primary vaccine failures (PVF)] [21].Seronegativity or vaccine failure may occur either because the immune response never developed or because it weaned over time [22]. Changes in the age of vaccination and type ofvaccine 
used over past decades may impact on future epidemiology of measles through differing rates of waning immunity [23].It had been found that $40 \%$ of children who had received 2 doses of measles vaccine (first dose before 12 months of age) were seronegative, and $33 \%$ of the seronegative ones did not respond to a third dose of vaccine [13]. While seroconversion rates in children after the first and second doses of measles vaccination at 9 and 15 months ofseroconvertion rates of $77.6 \%$ and $69.9 \%$ after the first dose and $81.9 \%$ and $90.3 \%$ after the second dose of measles vaccine [24].The SVF phenomenon due to waning immunity may become apparent only after the passage of years. It was suggested that vaccine-induced measles antibodies decline with time, and fall below protective levels [25].

In our study, after revaccination, $64.4 \%$ of 45 participants who were seronegative showed IgG \& IgM antibody response to revaccination. However there was no significant increase in antibody level postrevaccination in the 144 seropositive participants we examined ( $\mathrm{P}=0.577)$. These results were comparable with the finding of others elsewhere: Cohen and his colleagues found that $12 \%$ of seronegative individuals10-30years of age never developed a positive measles specific IgG titer after revaccination, while $58 \%$ did that remained positive for at least one year after vaccination [26]. Other study showed that $19.2 \%$ of people vaccinated were seronegative 411 years after the primary series of vaccination and $18.5 \%$ of seronegative individuals remainedseronegative after revaccination [27]. Other studies showed thatPeople with protective levels of antibodies did not respond torevaccination anammnestically, butseronegative individuals did [18, 28].To determines whether the seronegativity had been induced by PVF or SVF, the IgM antibody responses were assayed. Seven of 45 $(15.6 \%)$ seronegative individuals showed IgM antibody response afterrevaccination, the results suggested that they probably were a result ofPVF. The lack of an IgM response in other responders suggests the previous response to vaccination had been lost over time as have been suggested previously [29].

Recent successes in interrupting measles transmission in the World Health Organization Region of the Americas, most other countries in Europe and selected countries in other regions provide evidence for the feasibility of global eradication [30]. The World Health Organization has developed a global plan for accelerated measles control which calls for implementation of a strategy based on that used to successfully control measles in the Pan American Health Organization (PAHO): a catch-up campaign providing measles vaccine to all children regardless of prior history of immunization or disease, followed by high levels of routine coverage with measles immunization (keep-up) and periodic .Mass "catch up" measles immunization campaigns are recommended by the WHO asone of the main strategies to control or eliminate measles [31].To prevent measles outbreaks we have to have at least a coverage rate of $95 \%$ with measles vaccine [32].To interrupt virus transmission in a community, $>95 \%$ of a population must be protected. It seems impossible to reach a sufficiently high level of protection by routine vaccination. In this study among young adults in Iraq 17-24 years after scheduled measles vaccination, PVF and SVF were found to be $3.7 \%$ and $11.6 \%$ respectively. After revaccination, $85.1 \%$ of those vaccinated developed immunity. 
Considering the vaccine coverage of $95 \%$ and efficacy of $85 \%$, only $80.9 \%$ of individuals would be immune, a rate which may not prevent measles outbreaks. Un-immunized young children are at highest risk for measles and its complications, including death. However, any person who has not been immunized with vaccine or through experiencing the disease can become infected [21].Measles can be particularly deadly in countries experiencing or recovering from war, civil strife or a natural disaster. Infection rates increase because damage to infrastructure and health services interrupts routine immunization and overcrowding in camps for refugees and internally displaced people greatly increases the risk of infection [16].The geopolitical situation of the Region is complex: several countries are in a state of crisis and conflict or have experienced natural disasters, including Afghanistan, Iraq, and Pakistan. According to UNICEF, about 332 cases of measles have been reported since December 2012 in the Domiz refugee camp in Iraq [33]; so AMMAN/GENEVA, 30 April 2013 UNICEF and partners have stepped up vaccination campaigns in Syria, Jordan, Lebanon, Iraq and Turkey amid a number of measles outbreaks in a region already struggling to provide humanitarian assistance to millions of people affected by the Syrian crisis.Meanwhile, in Iraq's Domiz camp, about 19,300 refugees from the age of six months to 30 years were vaccinated with the support of UNICEF[34]. It is imperative to evaluate the vaccine with reduced potency because of poor storage and transportation, facilitated by the adverse effect of tropical climate on the vaccine [35]. Mulholland stated that; a generation of young adults with waning vaccine-derived immunity may become susceptible to some extent to wild measles infections, although the role of such "secondary vaccine failures" in measles epidemiology is unclear. The longer a community goes without circulating measlesvirus, the more vigilant public health officials must be to maintain immunity levels in the community [36].We concluded that; basing on the results of this and previous studies on measles epidemiology in Iraq [30, 37], and also taking into account experiences on measles control in other parts of the world [30, 31, 32, 38], we advise the implementation of World Health Organization-recommended strategies to reduce and interrupt indigenous measles virus transmission in Iraq. Mass vaccination with a keep-up phase and follow-up cyclic campaigns would reduce the number of susceptible individuals and to prevent new outbreaks. Additional SIAs are likely required to eliminate measles in Iraq.

\section{References:}

[1]WHO,1999. Manual for the laboratory diagnosis of measles viral infection.

[2]Schoenstadt, A., 2007.History of measles.

[3]CDC. Measles Imported by Returning U.S. Travelers Aged 6-23 Months, 2001-2011. MMWR. April 8, 2011 / 60(13); 397-400.

[4]WHO, 2008. Weekly feedback on measles in Iraq, immu accessed at Dec. 2008

[5]World Health Organization Global reductions in measles mortality 2000-2008 and the risk of measles resurgence. WklyEpidemiol Rec 2009; 84:509-16

[6] World Health Organization, "Weekly Feedback on Measles in Iraq," WHO Representative's Office in Iraq, 3/8/09 
[7]Ahmad S.,2012. Anbar project aims to conserve winter rainfall for future use.

[8]University of Nebraska Medical Center, College of Public Health, NE 68198-4355, USAjagar.

[9]WHO, 2008. Weekly Epidemiological records. Progress toward reducing measles mortality and eliminating measles, WHO EasternMediterranean Region 19972007, No.11

[10] Wilkins J. and, Wehrle RF. (1979); Additional evidence against measles vaccine administration to infants less than 12 months of age: altered immune response following active/passive immunization. Journal of pediatrics, 94(6):865-9.

[11]Redd SC, Markowitz LE, and Katz SL. (2003) Measles vaccine. In: Plotkin SA, Orenstein WA, eds. Vaccines, 3rd ed. Philadelphia, WB Saunders, 1999:222-66.

[12] Measles. In: Pickering LK, ed. Red book: 2003 Report of the Committee on Infectious Diseases, 26th ed. ELK Grove Village, American Academy of Pediatrics,:419-29.

[13]Linnemann CC Jr, Dine MS, Roselle GA and, Askey PA (1982)Measles immunity after revaccination: results in children vaccinated before 10 months of age. Pediatrics, 69(3):332-5.

[14] Expanded Programme on Immunization, Global Advisory Group. Weekly epidemiological record, 1981, 56(2):9-16.

[15]Hasony HJ,Thewaini HT and ,AlThamery AA(2002); Pattern of measles antibody responses fallowing vaccination in Basrah/southern

Iraq.J.BasicMed.Sc., 2(1); 62-67

[16]Sana'a H. Abdul Sahib and, Nazar A Mahmud (2011). Measles in KirkukGovernorate: Cohort Study.
Iraqi National Journal of Nursing specialties, vol.24, Issue (2)

[17]World Health Organization. Measles vaccines: WHO position paper WklyEpidemiol Rec 2009; 84:349-60.

[18] Saffar M.J., Alraza-Amiri M., Ajami A., Baba-Mahmoodi F., Khalilian A.R., Vahidshahi C. and, Shamsizadeh A. (2006). Measles seroepidemiology among adolescents and young adults: response to revaccination Volume 12 (5); 573-81

[19]Christina Poethko-Müller' and, Annette M. (2011) ; Seroepidemiology of measles-specific IgG antibodies and predictive factors for low or missing titers in a German population-based crosssectional study in children and adolescents (KiGGS)Vaccine ,Volume 29, Issue 45, 19 October, 7949-59.

[20] Chen RT, Markowitz LE,Albrecht Stewart JA,Mofenson LM,PrebulbSR and, Orenstein WA (1990); Measles antibody; reevaluation of protective titers. Journal of infectious diseases, 162(5); 1036-42

[21] WHO 2003 WHO recommended standards for surveillance of selected vaccines preventable diseases. WHO/V\&B/03.01

[22] ReddScMarkowits LE and, Katz SL.(1999): Measles vaccine. In: PlotkinSA,OrensteinWA,ed.

Vaccines, $3^{\text {rd }}$. ed. Philadelphia,WB Saunders, 222-66

[23]Nates, SV; Giordano, MO; Medeot, SI; Martinez, LC; Baudagna, AM; Naretto, E; Garrido, P; De Wolff, CD; (1998) Loss of maternally derived measles immunity in Argentinian infants. Pediatr Infect Dis J; 17:313-6. CrossRefMedline

[24] Ceyhan M, Kanra G, Erdem G and, Kanra B: (2001) 
Immunogenicity and efficacy of one dose measles-mumps-rubella (MMR) vaccine at twelve months of age as compared to monovalent measles vaccination at nine months followed by MMR revaccination at fifteen months of age. Vaccine, 19(31):4473-4478.

[25]Christensen B and, Bottiger M ( 1994),Measles antibody; comparison of long-term vaccination titers,early vaccination titers and naturally acquired immunity to and booster effects on the measles virus. Vaccine,12(2); 129-33

[26] Cohn ML, Robinson ED, Faerber M, Thomas D, Geyer S, Peters S, Martin M, Martin A, Sobel D and, Jones R.,( 1994),Measles vaccine failure: lack of sustained measlesspecific immunoglobulin G responses in revaccinated adolescents and young adults. PIDJ 1 13(1): 34-8.

[27] Poland GA, Jacobson RM, Thampy AM, Colbourne SA, Wollan PC, Lipsky JJ and, Jacobsen SJ.(1997)Measles reimmunization in children seronegative after initial immunization.JAMA. 277(14):1156-8.

[28]Davidkin I and, Valle M. (1998).Vaccine-induced measles virus antibodies after two doses of combined measles, mumps and rubella vaccine: a 12-yearfollow-up in two cohorts. Vaccine 16(20):2052-7.

[29] Wilkins J and, Wehrle RF, (1979) Additional evidence against measles vaccine administration to infants less than 12 months of age: altered immune response following active/passive immunization. JOP 94(6):865-9.

[30]De Quadros CA, (2004) Can measles be eradicated globally? Bulletin of the World Health Organization 82(2):134-8.

[31] Shuk KC, Yu LL and, Wei LL.(2002) "Mass Measles Immunization Campaign:

Experience in the Hong Kong Special Administrative Region of China". WHO; 80 (7), 585-591.

[32] Duke T, Mgone CS.(2003). "Measles not Just another Viral Exanthema". The Lancet, 361, 76371

[33]Syrian refugees straining health services in region, UN wa

[34] UNICEF. ORG (2013).Press release; Mass vaccination campaigns in Syria, Jordan, Lebanon, Iraq and Turkey amid measles outbreaks

[35] WHO (2000). Principles for Measles control and Elimination.

[36]Mulholland, E.K., (2006).Measles in the United States, 2006 Perspective;355 (5):440-3

[37] Abdul-Wahid SS (2008) Seroconversion rate after Measles vaccine in a selected sample of children under two years of age in Baquba city.Ph.D.thesis submitted to the college of Medicine AlMustansiryah University \Baghdad, Iraq.

[38]de Quadros CA, Hersh BS, Nogueira AC, Carrasco PA and, da Silveira CM (1998); Measles eradication: experience in the Americas. WHO, 76(suppl. 2):4752. 
دراسة مصلية وبائية لفيروس الحصبة بين اليافعين: الاستجابةلإعادة التطعيم

$$
\text { اروى هادى الحمدانسي* }
$$

*قسم الاحياء المجهرية/ كلية الطب مستتصرية.

\begin{abstract}
الخلاصة: الخام
قام الباحث بتقييم معدل الانتشار المصلي لمستضدات الحصبة والاستجابة لإعادة التمنيع (الحملة الوطنية الثاملة

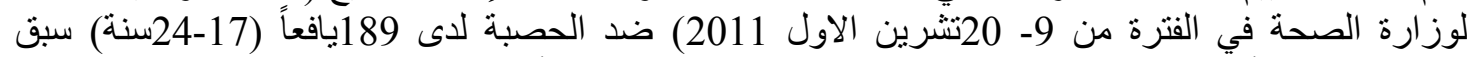

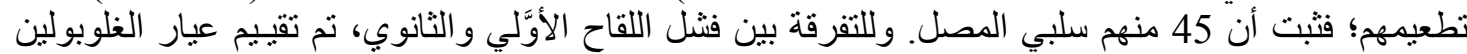

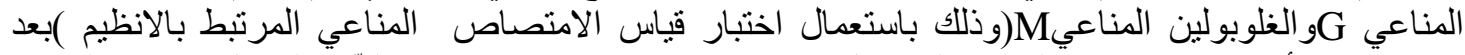

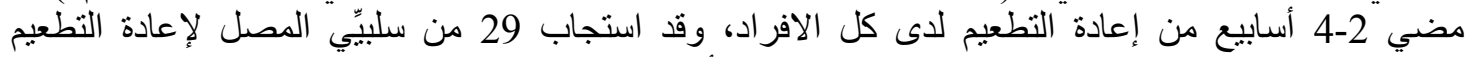

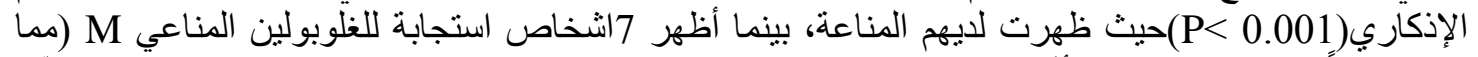

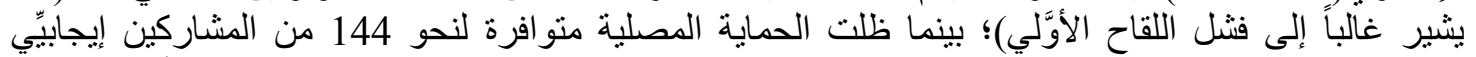

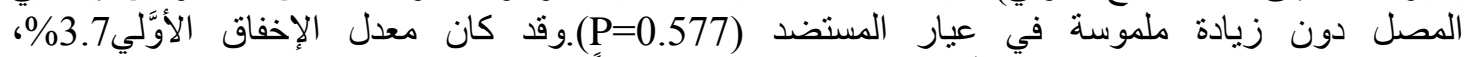

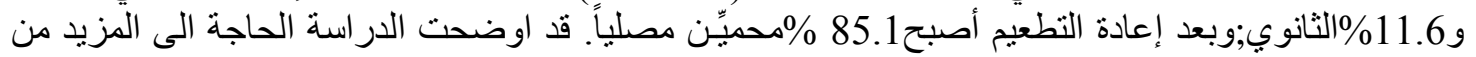

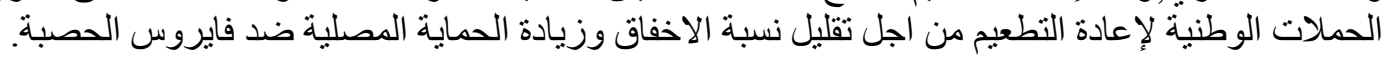

